\title{
Feasibility Study of a Neutron Activation System for EU Test Blanket Systems
}

\author{
Kuo Tiana,*, Pattrick Calderonipb, Bradut-Eugen Ghidersa”, and Axel Klixa \\ alnstitute for Neutron Physics and Reactor Technology, Karlsruhe Institute of Technology (KIT), Karlsruhe, Germany

\section{${ }^{b}$ Fusion for Energy(F4E), Barcelona, Spain}

The Neutron Activation System (NAS) for EU Helium Cooled Lithium Lead (HCLL) and Helium Cooled Pebble Bed (HCPB) Test Blanket Systems (TBS) is an instrument to determine absolute neutron fluence and absolute neutron flux with information on the neutron spectrum in Test Blanket Modules (TBMs).

- Functional Flow

- Activation probes are sent to Irradiation Ends (IEs) which is inside the TBMs

- Probes are exposed to neutron irradiation (from several tens of seconds up to a full plasma pulse)

- Probes are sent back to a counting station

- Induced gamma activities are subsequently measured by gamma-ray detector in the counting station

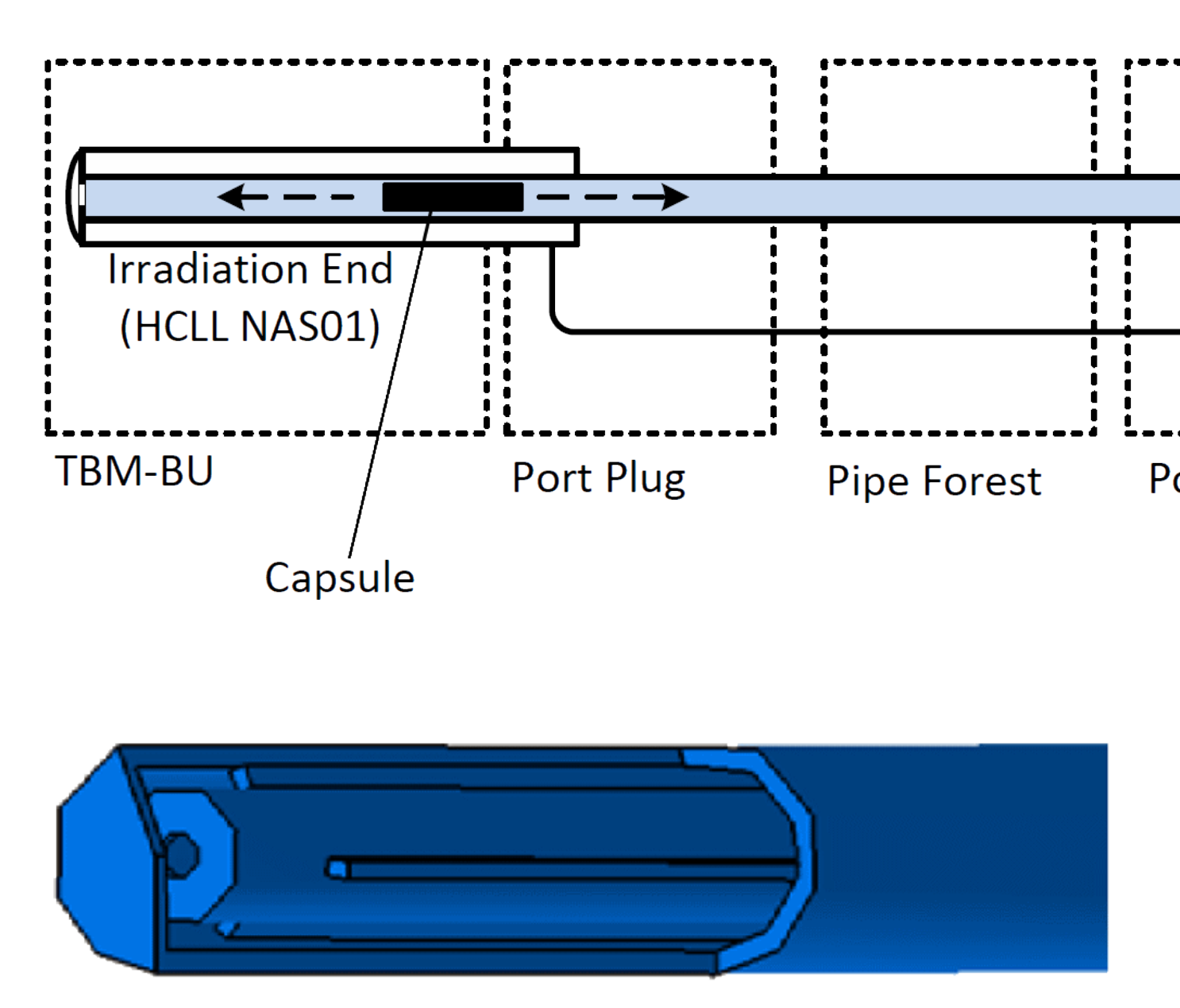

Irradiation End

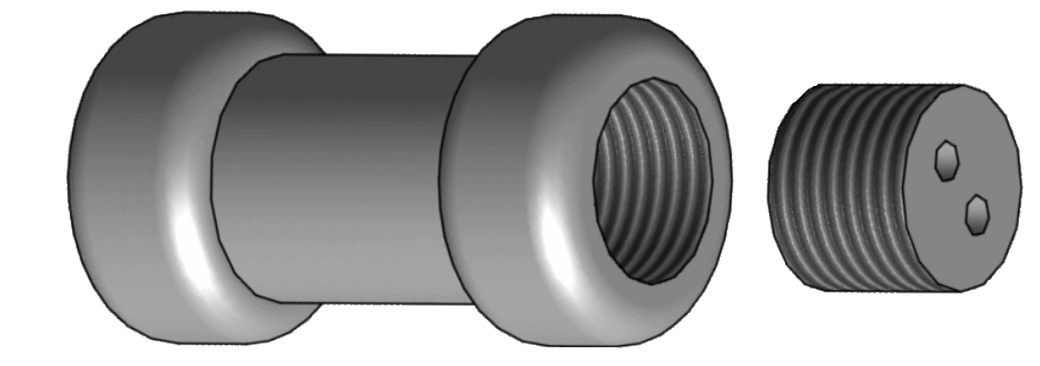

Capsule

\section{- Irradiation Ends}

- Coaxial double-walled structure

- With an opening hole in the end of the inner pipe

- Inner pipe connects transfer pipe

- Outer pipe connects return gas pipe

- Installed inside TBM BUs

- No bending before penetrating all BPs of the TBMs

- Material: EUROFER
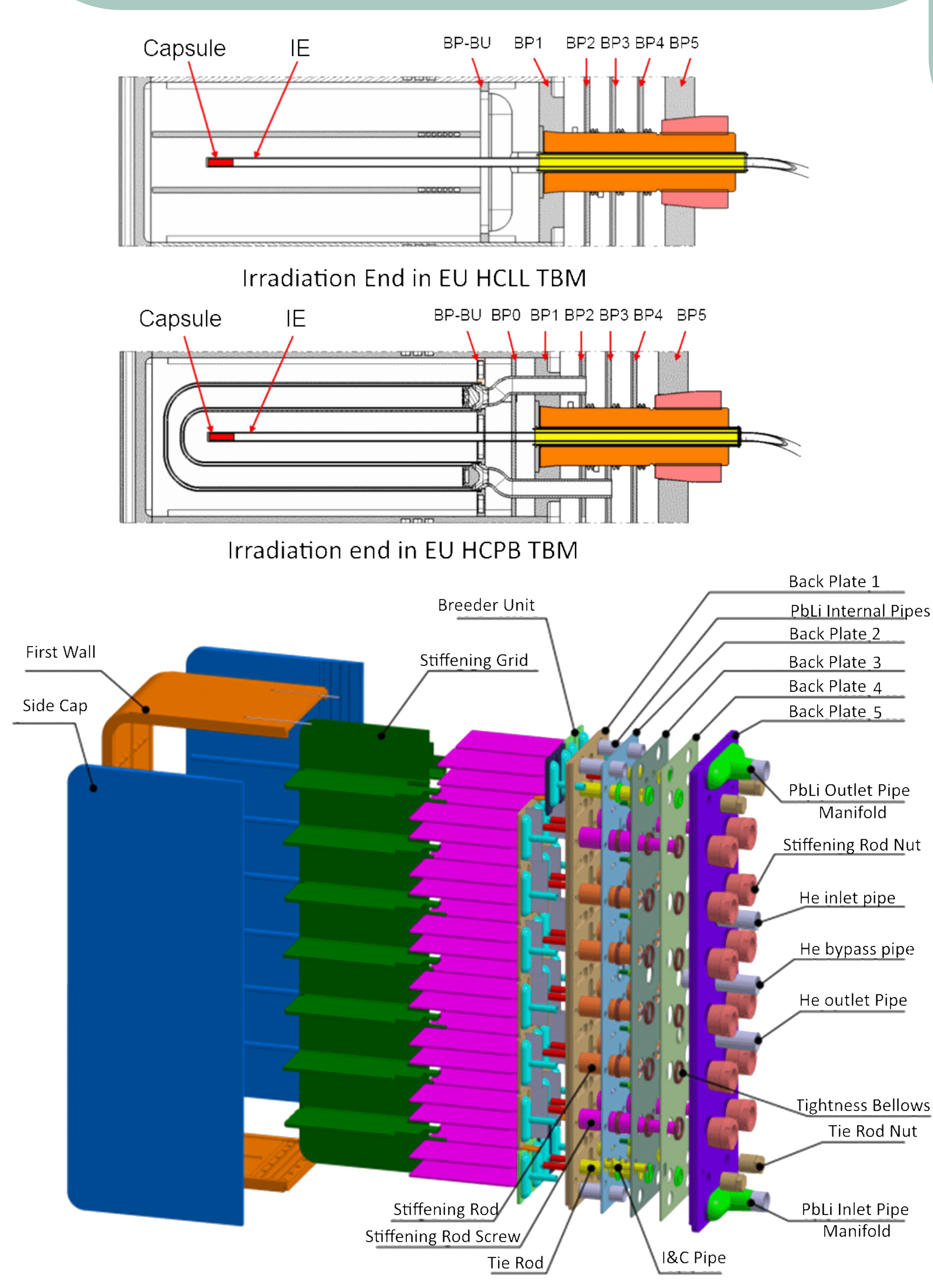

- Capsule

- Made of CFC (Carbon fiber-reinforced carbon) or Niobium

- Cylindrical shape ext. diameter: $8 \mathrm{~mm}$

- With a threaded bore of $5 \mathrm{~mm}$ in diameter

- Activation materials filled in the bore

- The capsule itself can also be activatio sample

- Integration of NAS to TBS

- IEs have to penetrates all back plates of the TBMs

- The hollowed stiffening rods will be used to accommodate the IEs (and other diagnostic cables/lines)

- Transfer pipes and return gas pipes penetrate all of the interfaces between TBMs and Tritium building

- Challenges

- Active cooling for IEs may be required in HCPB TBM: environment temperature can reach up to $650^{\circ} \mathrm{C}$

- Limited space in instrumentation penetration pipes: NAS occupies $17 \%$ cross section area reserved for all diagnostic lines
- Key Specifications and Components

- HCLL and HCPB TBM have respective independent NAS

- For each TBM, three NAS IEs are arranged

- HCLL and HCPB TBMs share one common counting station, including gamma-ray detectors

- Capsule loader for distributing capsules to transfer pipes

- Pneumatic transfer system for sending capsules to IE and retrieving capsules

- Capsule positions are monitored using optical fibers

- Transfer Pipes

- Inner diameter : 9 mm

- Outer diameter: $12 \mathrm{~mm}$

- Material: 316L stainless steel

- Return Gas Pipes

- Inner diameter : 6 mm

- Outer diameter: $4 \mathrm{~mm}$

- Material: 316L stainless steel

- Pressure difference between the two pipes drive capsules between IE and transfer station

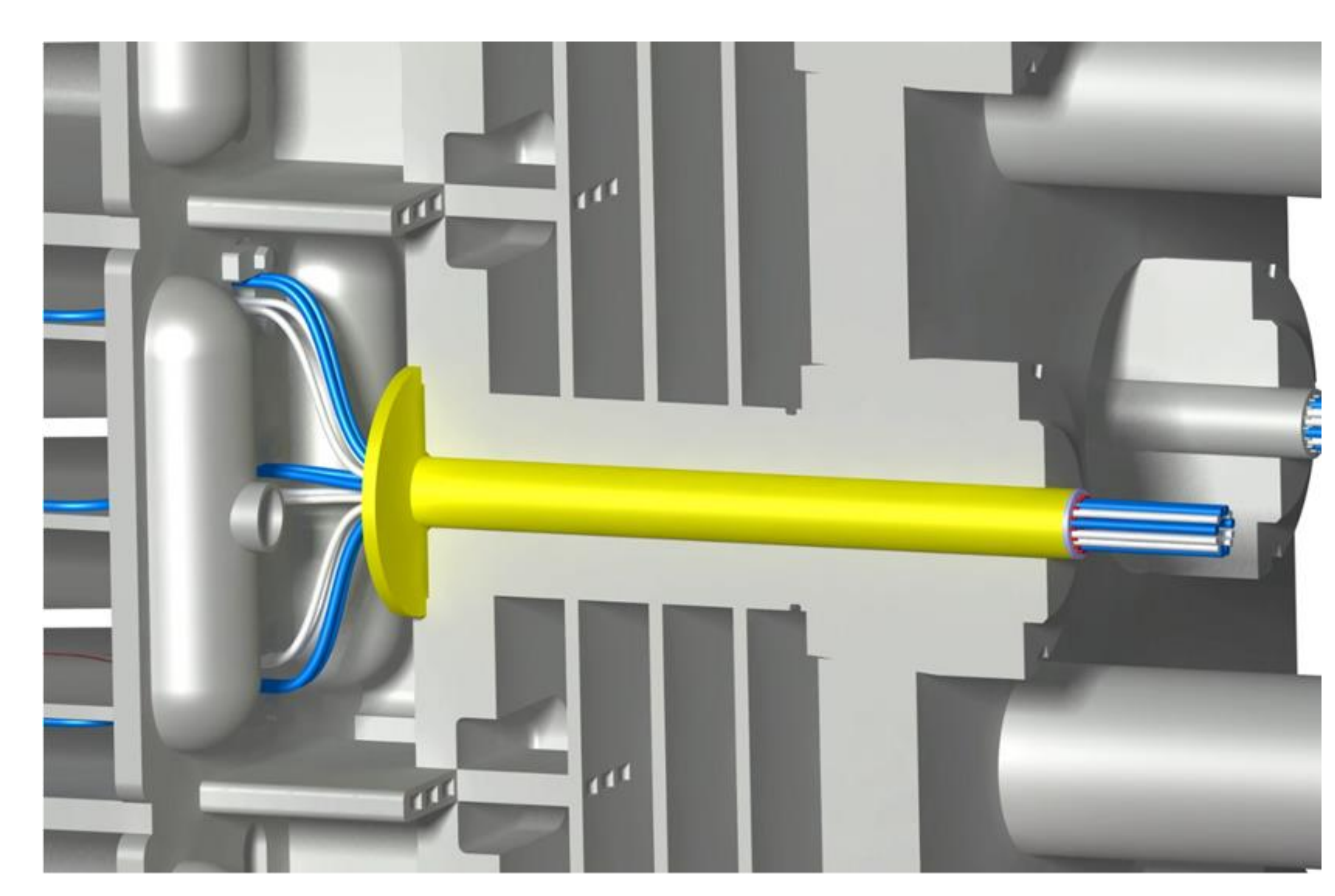

*Corresponding author: kuo.tian@kit.edu 